Original Article

\title{
The Control of Postural Stability during Standing is Decreased in Stroke Patients during Active Head Rotation
}

\author{
Tsubasa Mitsutake, PT ${ }^{1,2)^{*}}$, Yuji Chuda, $\mathrm{PT}^{1)}$, Shinichiro OKa, $\mathrm{PT}^{3)}$, Hirokatsu Hirata, PT ${ }^{4}$, \\ Takashi Matsuo, OT $^{1)}$, Etsuo Horikawa, PhD $^{2)}$ \\ 1) Department of Rehabilitation, Shiroishi Kyoritsu Hospital, Japan \\ 2) Division of Cognitive Neuropsychology, Graduate School of Medicine, Saga University: 1296 \\ Hukuta, Shiroishi, Kishima, Saga 849-1112, Japan \\ 3) Department of Physical Therapy, School of Health Sciences at Fukuoka, International University of \\ Health and Welfare, Japan \\ 4) Department of Rehabilitation, Takagi Hospital, Japan
}

\begin{abstract}
Purpose] The aim of this study was to evaluate the effect of active head rotation on postural control in stroke patients during standing as compared with age-matched healthy subjects. [Subjects and Methods] In total, 46 stroke patients and 37 age-matched healthy subjects were recruited for the study. A stabilometer was used to assess postural stability in participants during standing, with or without active head rotation, and with their eyes open or closed. Subjects were asked to stand on a force plate while rotating their head in the yaw plane at a frequency of $1.0 \mathrm{~Hz}$. A metronome was used to maintain the head rotation frequency, and the head rotation range was maintained at a total of $70^{\circ}$ during the postural stability examinations. [Results] The control of postural stability during standing with active head rotation was significantly decreased in the stroke group as compared with the healthy group with both the eyes open and closed. No significant differences in relation to standing without head motion were observed between groups. [Conclusion] The findings suggest that postural instability is increased in stroke patients during active head rotation, and therefore, vestibular function in relation to head rotation might be reduced in stroke patients.

Key words: Stroke patients, Postural stability, Vestibular function
\end{abstract}

(This article was submitted Mar. 14, 2014, and was accepted May 21, 2014)

\section{INTRODUCTION}

As components of the sensory system of the body, the visual, vestibular, and somatosensory senses play an important role in postural stability ${ }^{1,2)}$. The proper coordination of these senses in relation to changes in the immediate environment helps prevent falls, and the impairment of this coordination can adversely affect the control of posture, thus increasing the risk of falls. In particular, studies on neurological diseases have indicated that strokes increase the risk of falls because of various impairments in the motor and high cerebral functions ${ }^{3)}$. As a potential compensatory mechanism for these impairments, stroke patients tend to rely more on visual sensory information than vestibular or somatosensory information in the sensory system ${ }^{4}$. However, paradoxically, the acquisition of important visual information can increase the occurrence of falls, because

*Corresponding author. Tsubasa Mitsutake (E-mail: mitutuba1012@gmail.com)

(C2014 The Society of Physical Therapy Science. Published by IPEC Inc. This is an open-access article distributed under the terms of the Creative Commons Attribution Non-Commercial No Derivatives (by-ncnd) License $<$ http://creativecommons.org/licenses/by-nc-nd/3.0/> . rapid head movement can cause retinal slip and degrade visual acuity ${ }^{5}$. In a previous study, even healthy subjects were shown to lose postural stability during active head movements ${ }^{6}$. However, this postural perturbation due to head movements is further increased in stroke patients. Therefore, stroke patients may not adequately coordinate their postural control with head movement, which might result in a high risk of falling as compared with healthy subjects. The aim of the present study was to evaluate the effect of active head rotation on postural control in stroke patients while they stood as compared with age-matched healthy subjects.

\section{SUBJECTS AND METHODS}

This study was approved by a local ethics committee, and subjects provided written informed consent. Stroke patients were enrolled in the study if they (1) had experienced their first hemorrhagic or ischemic stroke within the past 6 months (from stroke onset) and (2) had the ability to stand barefoot in an upright position with both feet together on a platform without support and with eyes closed for more than 60 seconds. Based on these inclusion criteria, a total of 46 stroke patients were enrolled in this study. Lesions 
Table 1. Demographics and experimental results of the stroke patients and healthy control subjects

\begin{tabular}{lcc}
\hline & Control subjects $(\mathrm{n}=37)$ & Stroke patients $(\mathrm{n}=46)$ \\
\hline Age (years) & $67.97 \pm 10.78$ & $70.04 \pm 8.35$ \\
Gender (male/female) & $21 / 16$ & $29 / 17$ \\
Stroke classification (ischemic/hemorrhagic) & - & $35 / 11$ \\
Hemiparesis (right/left) & - & $23 / 23$ \\
Time since stroke onset (days) & - & $37.35 \pm 37.24$ \\
NIHSS (score) & - & $2.18 \pm 1.86$ \\
FMA-LL (score) & - & $30.20 \pm 3.97$ \\
FIM (score) & & $116.09 \pm 9.61$ \\
Static standing (cm) & $99.73 \pm 32.82(60.55-227.39)$ & $100.63 \pm 41.66(28.45-206.65)$ \\
Eyes open & $134.70 \pm 52.88(65.84-342.88)$ & $139.23 \pm 64.20(29.61-337.23)$ \\
Eyes closed & & $165.79 \pm 82.36(50.48-439.61)^{*}$ \\
Standing during active head rotation (cm) & $132.65 \pm 49.81(66.62-281.10)$ & $230.62 \pm 104.01(67.73-502.49)^{*}$ \\
$\quad$ Eyes open & $182.16 \pm 100.11(35.16-592.90)$ & \\
Eyes closed & & \\
Mean \pm SD (range); $*$ p $<0.05$ & &
\end{tabular}

encompassed the following cerebral areas of the stroke patients: brain stem $(19.6 \%)$, putamen $(15.2 \%)$, thalamus $(13.0 \%)$, frontal lobe $(10.9 \%)$, parietal lobe $(8.7 \%)$, temporal lobe $(8.7 \%)$, cerebellum $(8.7 \%)$, occipital lobe $(6.5 \%)$, corona radiata $(6.5 \%)$, and basal ganglia $(2.2 \%)$. The control group consisted of 37 age-matched healthy subjects.

Postural stability was examined using a stabilometer (G620; Anima Corp., Tokyo, Japan). Each subject was asked to stand barefoot in an upright position with both feet together on a platform and with both the eyes open or closed for 60 seconds. In the eye-open condition, the subjects' eyes were fixated on a target $(1.0 \mathrm{~cm}$ in diameter $)$, which was positioned at the same height as the subjects' eyes. In the eye-open and eye-closed conditions, postural control was measured in the participants while they stood without head motion (static standing) or with active head rotation. Postural control during static standing was measured with subjects standing quietly on a force plate. Moreover, postural control during active head rotation was measured with subjects standing on the force plate while rotating their head in the yaw plane at a frequency of $1.0 \mathrm{~Hz}$. A metronome was used to maintain the head rotation frequency, and the head rotation range was maintained at a total of $70^{\circ}$ during the postural stability examinations. During the examination, subjects were requested not to compensate for their movement with trunk movement. The measurements were repeated if trunk movement occurred. The locus length (LNG) was used for evaluating the stroke and control groups and was calculated as the total movement distance from the center of pressure.

All statistical analyses were performed using SPSS version 19 (IBM, Armonk, NY, USA), and the level of statistical significance was set at 0.05 . The Shapiro-Wilk test revealed the data were not normally distributed; therefore, the Mann-Whitney U test was used to compare the values of the stroke patients with those of the healthy subjects.

\section{RESULTS}

No significant differences in postural control during static standing were observed between the study groups. However, the control of postural stability during standing with active head rotation was significantly decreased in the stroke group as compared with the healthy group for both the eye-open and eye-closed conditions $(\mathrm{p}<0.05)$ (Table 1$)$.

\section{DISCUSSION}

In the present study, significant postural instability was observed in the stroke patients during standing with active head rotation as compared with age-matched healthy subjects in both the eye-open and eye-closed conditions. Previous research has suggested that the sensory system for postural control stabilizes the visual, vestibular, and somatosensory information to achieve consistency ${ }^{7)}$. In those with sensory deficits, rapid head movements may cause retinal slip and degradation of visual acuity ${ }^{5)}$. During these movements, the sensory strategy allows for compensation of visual function via vestibular and somatosensory information. In particular, the vestibular sensory regions experience increased activation due to head motion ${ }^{8}$, which contributes to postural stability. A previous study has shown that vestibular function is involved in maintaining postural stability during head rotation ${ }^{9}$, and the methods of the present study could have activated vestibular sensory function. However, many patients with hemiplegia cannot adequately reduce their weight to utilize vestibular information and rely on visual input. Thus, the vestibular senses of stroke patients are decreased as compared with those of healthy subjects, which can affect their postural stability during standing with head motion in both the eye-open and eye-closed conditions. Stroke damages a part of the neural network of the affected hemisphere and consequently can impact not only motor function but also the vestibular and 
somatosensory senses. Extensive anatomical connections are present between the cerebral cortex and the vestibular system. Of interest, in a functional magnetic resonance imaging study of the multisensory cortical signal changes occurring during vestibular galvanic stimulation, the frontal cortex, parietal cortex, thalamus, putamen, and parietoinsular vestibular cortex were found to be active ${ }^{10)}$. In the present study, many stroke patients showed impairment of these cerebral regions, which might have been related to the reduced ability for postural control.

This study had several limitations. It was difficult to conclude whether the area of the injured brain affected the postural stability of the patients. Furthermore, in the evaluation of postural control, visual acuity was not evaluated. In future studies, the cerebral activation during head rotation should be considered, and the effect of visual acuity should be assessed using a dynamic visual acuity test.

\section{REFERENCES}

1) Shumway-Cook A, Woollacott MH: Motor Control: translating research into clinical practice, 4 th ed. Philadelphia: Lippincott Williams \& Wilkins, 2012, pp 161-194.
2) Park YH, Kim YM, Lee BH: An ankle proprioceptive control program improves balance, gait ability of chronic stroke patients. J Phys Ther Sci, 2013, 25: 1321-1324. [Medline] [CrossRef]

3) Thurman DJ, Stevens JA, Rao JK, Quality Standards Subcommittee of the American Academy of Neurology: Practice parameter: assessing patients in a neurology practice for risk of falls (an evidence-based review): report of the Quality Standards Subcommittee of the American Academy of Neurology. Neurology, 2008, 70: 473-479. [Medline] [CrossRef]

4) Bonan IV, Marquer A, Eskiizmirliler S, et al.: Sensory reweighting in controls and stroke patients. Clin Neurophysiol, 2013, 124: 713-722. [Medline] [CrossRef]

5) Tian JR, Shubayev I, Demer JL: Dynamic visual acuity during transient and sinusoidal yaw rotation in normal and unilaterally vestibulopathic humans. Exp Brain Res, 2001, 137: 12-25. [Medline] [CrossRef]

6) Morimoto H, Asai Y, Johnson EG, et al.: Effect of oculo-motor and gaze stability exercises on postural stability and dynamic visual acuity in healthy young adults. Gait Posture, 2011, 33: 600-603. [Medline] [CrossRef]

7) Naito Y, Tateya I, Hirano S, et al.: Cortical correlates of vestibulo-ocular reflex modulation: a PET study. Brain, 2003, 126: 1562-1578. [Medline] [CrossRef]

8) Dieterich M: Central vestibular disorders. J Neurol, 2007, 254: 559-568. [Medline] [CrossRef]

9) Ward BK, Mohammed MT, Brach JS, et al.: Physical performance and a test of gaze stabilization in older adults. Otol Neurotol, 2010, 31: 168-172. [Medline] [CrossRef]

10) Bense S, Stephan T, Yousry TA, et al.: Multisensory cortical signal increases and decreases during vestibular galvanic stimulation (fMRI). J Neurophysiol, 2001, 85: 886-899. [Medline] 